\title{
Circuit
}

Musiques contemporaines

\section{Introduction : à la recherche des neumes de demain}

\section{Isabelle Panneton et Marie-Pierre Brasset}

Volume 25, numéro 1, 2015

Contenir le sonore : les nouveaux profils de la notation

URI : https://id.erudit.org/iderudit/1029472ar

DOI : https://doi.org/10.7202/1029472ar

Aller au sommaire du numéro

Éditeur(s)

Les Presses de l’Université de Montréal

ISSN

1183-1693 (imprimé)

1488-9692 (numérique)

Découvrir la revue

Citer ce document

Panneton, I. \& Brasset, M.-P. (2015). Introduction : à la recherche des neumes de demain. Circuit, 25(1), 5-7. https://doi.org/10.7202/1029472ar d'utilisation que vous pouvez consulter en ligne.

https://apropos.erudit.org/fr/usagers/politique-dutilisation/ 


\section{Introduction : à la recherche des neumes de demain}

Isabelle Panneton et Marie-Pierre Brasset

Dans son article intitulé «Avant de tourner la page », Georges Forget rappelle que les neumes utilisés pour le chant grégorien au IX siècle sont « de petits signes placés au-dessus du texte à chanter, indiquant les profils mélodiques ». Aujourd'hui, des signes graphiques de toutes sortes dessinent dans les partitions de nouveaux «profils» (densités, enveloppes, fluctuations d'énergie...). Ces traductions analogiques du sonore se situent aux deux extrêmes de l'évolution de la notation musicale, laquelle s'est échelonnée sur plus de dix siècles au service non seulement de la transmission des œuvres, mais aussi de leur élaboration et de leur complexification.

L'œuvre musicale a de tout temps débordé le cadre du système de notation, ce dernier laissant plus ou moins place, selon les époques, à l'interprétation. Ce constat est plus que jamais pertinent, alors que les territoires explorés se sont élargis et posent de nouveaux défis pour qui veut en tracer ou en préciser le parcours.

Rappelons que, dès la seconde moitié du $\mathrm{xx}^{\mathrm{e}}$ siècle, la partition traditionnelle s'enrichit de nouvelles conventions associées à l'élargissement des techniques et des modes de jeu. L'émergence des musiques électroacoustique, spectrale, mixte, de même que les nouvelles interactions entre compositeurs et interprètes rendues possibles grâce aux outils numériques ont forcé l'exploration de nouveaux systèmes de notation.

Chercheurs et théoriciens s'intéressent actuellement à la transformation et aux nouveaux enjeux de la notation musicale, comme en témoignent de nombreux événements nationaux et internationaux. Ainsi, une journée d'étude (Séminaire notation) a été organisée par l'AFIm/INA-GRM en février 2015ํ. Du 7 au 9 mai 2015, l'Université de Montréal organisera pour la première fois en Amérique du Nord les Journées d'informatique musicale (JıM)² avec le
1. Voir: <http://notation.afim-asso org/doku.php/evenements/2015-02o6-etude-notation3> (consulté le 23 février 2015). 2. Voir: <http://jim2015.oicrm.org> (consulté le 23 février 2015). 
3. Voir: <http://music-encoding.org/ conference $>$

(consulté le 23 février 2015).

4. Voir: <http://tenor2015.tenorconference.org $>$

(consulté le 23 février 2015).

5. Voir: <http://cmr.soc.plymouth. ac.uk/cmmr2015>

(consulté le 23 février 2015). soutien de l'Observatoire interdisciplinaire de création et de recherche en musique (OICRM). Les 18-21 mai 2015, à Florence (Italie), se tiendra la Music Encoding Conference 3 . S'ajouteront encore TENOR 2015 (First International Conference on Technologies for Music Notation and Representation) ${ }^{4}$, les 28-30 mai 2015, à Paris, ainsi que le $11^{\text {th }}$ International Symposium on Computer Music Multidisciplinary Research (CMmR) Music, Mind, and Embodiment, les 16-19 juin 2015 à Plymouth (Angleterre)5.

Dans le dossier thématique que nous proposons, musicologues, compositeurs et interprètes partagent leurs réflexions à partir de leur propre espace de recherche. Ainsi, le volet historique, présenté par le compositeur Georges Forget, offre un survol des outils ayant servi à l'édition musicale au fil des siècles. Il rappelle ce que fut le métier de graveur, la profondeur de la formation musicale qu'exigeait cet art en voie de disparition. Il met en évidence que la notion de technologie n'est pas l'apanage de l'ordinateur et que la représentation musicale est parfois modelée par l'outil.

Le timbre, qui émane de nombreux facteurs en jeu dans la production du son, demeure difficile à traduire de façon précise dans le contexte du système de notation traditionnel. La musicologue Caroline Traube expose les stratégies adoptées par les compositeurs, «en amont ou en aval de la relation geste-son », pour agir sur ce paramètre multidimensionnel. Elle présente également un système de notation chinois qui renverse la hiérarchie occidentale en plaçant la notation du timbre au centre du système d'écriture.

Pierre Couprie explore la problématique très actuelle de la transcription des musiques électroacoustiques en posant d'entrée de jeu une question fondamentale: «Que cherchons-nous à démontrer ou à présenter? » Après un parcours critique des approches existantes, il propose un modèle de représentation de caractère modulaire qui intègre des pratiques relatives à l'analyse musicale et divers objets graphiques servant à la représentation et à l'analyse d'œuvres non notées.

François-Xavier Féron aborde pour sa part l'évolution du système de notation de Helmut Lachenmann dans Pression pour violoncelliste. Une nouvelle édition de l'œuvre originale (1972) a été réalisée en 2012 : à travers l'analyse d'extraits choisis, le musicologue découvre une partition hors normes, à la fois complexe et précise, qui s'attache à représenter les actions des interprètes plutôt que les sons produits par l'instrument; ce faisant, il met en évidence comment, au-delà des techniques et des modes de jeu nouveaux, Lachenmann arrive à créer un « univers sonore inouï».

Enfin, ce dossier thématique présente la retranscription d'entrevues que Marie-Pierre Brasset a menées avec trois chefs d'orchestre de la scène de la 
création musicale contemporaine montréalaise, soit Lorraine Vaillancourt, Véronique Lacroix et Walter Boudreau. Menées individuellement, ces entrevues dévoilent la nature particulière du lien qu'entretient chacun d'eux avec la notation. Ils ne manquent pas de souligner les écueils souvent rencontrés dans leur métier, mais ils expriment aussi leur admiration devant le haut degré de sophistication des systèmes de notation avec lesquels ils travaillent. Le numéro se conclue avec une Exclusivité web présentant le tout dernier balado (podcast) réalisé par Circuit, en partenariat avec la revue Intermédialités, et intitulé «L'autre Gould». La rubrique des Actualités offre ensuite un nouveau volet de la chronique «Entendu dans Cette ville étrange», rédigé par André Hamel, suivi de deux comptes rendus: l'un, de Cléo Palacio-Quintin, sur l'ouvrage Word Events: Perspectives on Verbal Notation de John Lely et James Saunders, l'autre, de Chantale Laplante, sur le collectif montréalais et étiquette de disques Kohlenstoff. Enfin, Symon Henry dresse un bilan du projet Génération 2014 de l'Ensemble contemporain de Montréal $(\mathrm{ECM}+)^{6}$.

Bonne lecture!

Montréal, février 2015
6. [ndlr] Première partie seulement. La suite paraîtra dans le prochain numéro de Circuit (vol. 25, ${ }^{\circ}{ }^{2}$ ). 